\title{
RESEARCH HIGHLIGHT Proteasome phase separation: a novel layer of quality control
}

\author{
Victoria Cohen-Kaplan ${ }^{1}$, Ido Livneh ${ }^{1}$ and Aaron Ciechanover ${ }^{1}$ \\ Cell Research (2020) 30:374-375; https://doi.org/10.1038/s41422-020-0306-9
}

\begin{abstract}
Maintenance of the quality control of the cellular proteome involves several pathways and a variety of transient and interchangeable assemblies. A recently discovered membraneless foci that contain - among several components - the 265 proteasome complex and that are generated in response to a proteotoxic stress, is the recent addition to this evergrowing list.
\end{abstract}

One of the fundamental problems in biology is how cells recruit and concentrate their 'divisions' of enzymes and reactants in order to respond to different environmental and internal stresses and cues. Eukaryotic cells contain numerous membrane-bound organelles - such as the nucleus, mitochondria, the endoplasmic reticulum and the Golgi apparatus, and lysosomes that carry out specific functions and regulate distinct cellular processes. However, cells can also amass transient supramolecular assemblies/ machineries which consist of different proteins, nucleic acids and other components, but lack separating membranes. The advantages of not having a 'fence' make these assemblies accessible to a variety of substrates of different characteristics that otherwise may need special transport mechanisms. It also enables these complexes to be rapidly assembled and disassembled, exchange their components in an efficient way according to arising needs, and rapidly mobilize the different constituents to different cellular sites as needs change. These membraneless compartments can be present in the nucleus (e.g., promyelocytic leukemia protein (PML) nuclear bodies (NB), Cajal bodies, the nucleolus, and nuclear speckles), as well as in the cytoplasm (e.g., P bodies, stress granules (SGs), processing bodies, and the centriole), and some of them are related to the proteasome. The appearance of nuclear/ cytosolic bodies or dysregulation of their structure and functions (as in the case of PML-NB) can underlie the pathogenesis of many diseases, including neurodegenerative disorders, inflammation and malignancies (e.g., promyelocytic leukemia). Many of these bodies have been discovered a while ago, however, their structure, mechanisms of biogenesis, and mostly functions, have started to emerge only recently. It has been shown that liquidliquid phase separation (LLPS) drives the formation and maintenance of these membraneless assemblies that possess liquid-like properties. Interestingly, formation of some of these assemblies requires the ubiquitin-proteasome system. ${ }^{1-3}$

In a study reported recently, Yasuda and colleagues ${ }^{4}$ discovered a new type of proteasome-containing nuclear foci, which are formed under acute hyperosmotic stress and do not co-localize with previously identified nuclear bodies, such as PML-NB and Cajal bodies. These proteasome-containing foci are transient, and consist - in addition to the $26 \mathrm{~S}$ proteasome - of ubiquitinated proteins, p97 and multiple proteasome-interacting proteins. It appears that their function is to serve as proteolytic centers.
Inhibition of proteasome activity results in an increased number and size of these foci, as well as in their delayed clearance. Furthermore, the researchers showed that these foci possess liquid droplet-like properties by demonstrating: (1) that they are specifically sensitive to 1,6-hexanediol which destabilizes such structures; (2) that small foci can fuse into larger ones; and (3) that the proteasome can be easily exchanged.

The proteasome is a large complex of $\sim 2.5 \mathrm{MDa}$, which consists of 33 'canonical' subunits arranged in two sub-complexes: the $20 \mathrm{~S}$ core particle (CP) and one or two 19S (or other) regulatory particles (RPs). ${ }^{5-7}$ The proteasome is the catalytic arm of the ubiquitin-proteasome system (UPS) and is responsible for the degradation of the majority of short-lived/regulatory proteins, as well as of misfolded/mutated/damaged proteins (following their covalent marking by ubiquitin). Yasuda and colleagues found that hyperosmotic stress affects ribosome biosynthesis as well as ribosomal protein assembly. This results in increased ubiquitination of orphan/unassembled ribosomal proteins (e.g., RPL15, PRL29 and RPS2) with their subsequent proteasomal degradation occurring at the newly described foci. Further, they found that formation of these foci is mediated by RAD23B, a Ub-associated (UBA)-Ub-like (UBL) shuttling protein, that binds ubiquitinated substrates via its C-terminal UBA domain and to the 19S RP ubiquitin receptors RPN1, RPN10 and RPN13 via its N-terminal UBL domain. ${ }^{8}$ Furthermore, the researchers demonstrated that the polyubiquitinated proteins are also required for LLPS of the proteasome in the presence of RAD23B: inhibition of the ubiquitin-activating enzyme, E1, almost completely prevented foci formation. They also found that formation of the foci depends on the UBA domain of RAD23B: reconstitution of RAD23B-KO cells with a mutated form of RAD23B which lacks its UBA domain, failed to induce LLPS of the proteasome. In agreement with the role of UBA-UBL proteins in proteasomal degradation, RAD23B was shown to preferentially bind K48-linked polyubiquitin chains. ${ }^{9}$ In the current study, the researchers showed that incubation of RAD23B and K48Ub chains in a cell-free system resulted in RAD23B's UBA domain-mediated formation of spherical condensates. They further showed that the formation of these spherical condensates depends not only on the concentration of each protein, but also on the length of the Ub chains. While only long K63-linked ubiquitin chains were able to form condensates with RAD23B, formation of K48-linked chain condensates could be driven by a short, tetra-ubiquitin chain.

The life cycle of the proteolytic foci is depicted in Fig. 1.

Recently, it was shown that another shuttling protein, p62/ SQSTM1, can promote polyubiquitin chain-induced LLPS in a UBA domain-dependent manner. ${ }^{3}$ Additionally, p62/SQSTM1 is known to deliver ubiquitinated substrates to the proteasome by

\footnotetext{
${ }^{1}$ Technion Integrated Cancer Center, The Rappaport Faculty of Medicine and Research Institute, Technion-Israel Institute of Technology, 3109602 Haifa, Israel
} Correspondence: Aaron Ciechanover (aaroncie@technion.ac.il)

Published online: 7 April 2020 


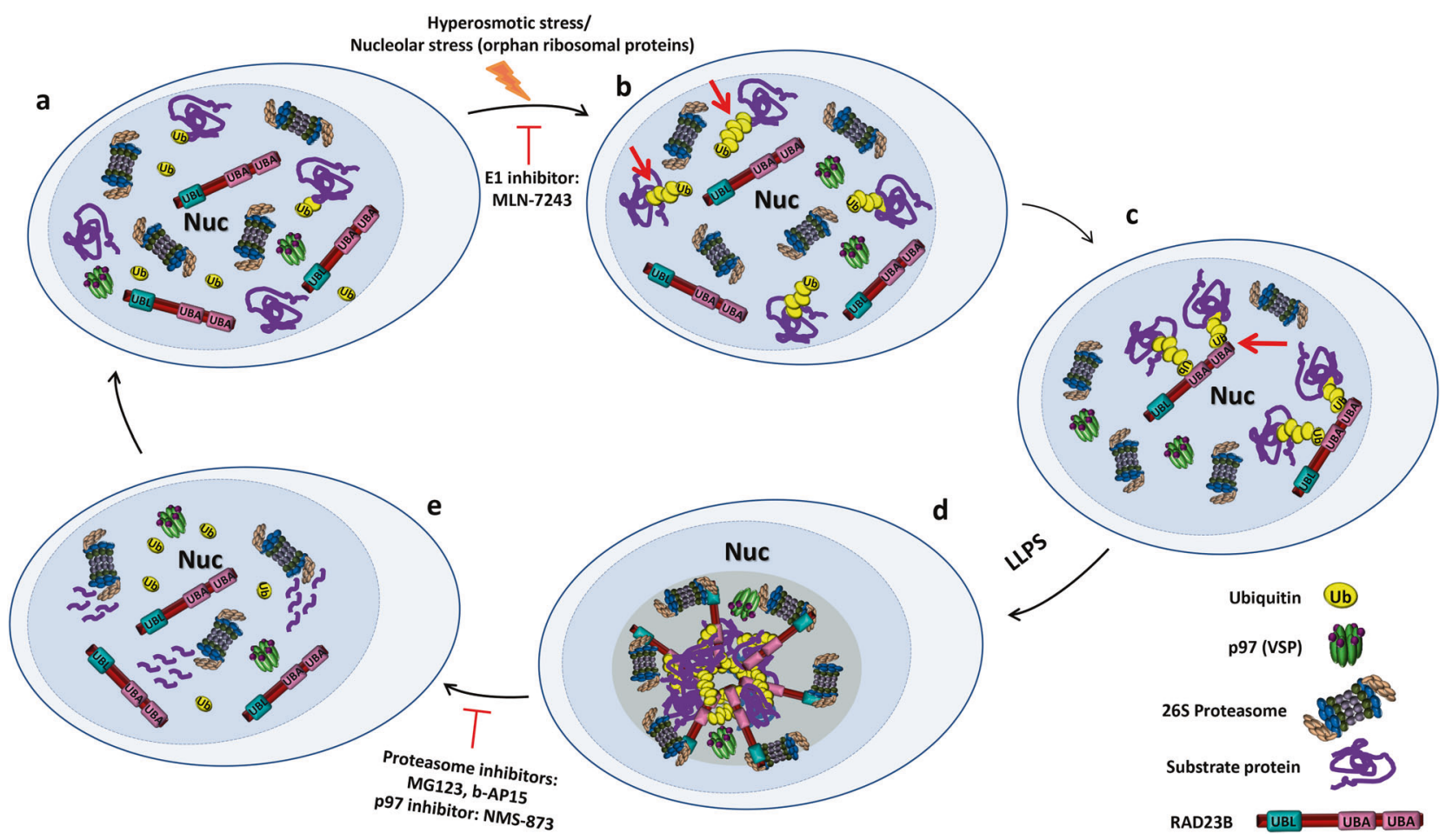

Fig. 1 Hyperosmotic stress induces polyubiquitination of faulty nuclear proteins along with RAD23B-mediated formation of proteolytic proteasome-containing foci. a Cell under normal conditions. b Following hyperosmotic stress, ubiquitination of orphan, unassembled ribosomal proteins and other nuclear proteins is upregulated. c The ubiquitinated nuclear proteins are recognized by the UBA domain of RAD23B that induces the formation of Ub-chains/RAD23B phase-separated condensates. d RAD23B recruits the proteasome to the condensates via its UBL domain. e The proteasome and p97 degrade the ubiquitinated proteins.

interacting with the RPN1 195 proteasomal subunit. ${ }^{10}$ These findings raise the question of whether p62 can also promote the formation of proteasomal foci in a ubiquitin chain-dependent manner.

In addition, p97 and the ubiquitin ligase UBE3A (E6-AP) were also identified as components of the stress-induced proteasomal foci. However, only p97 was found to affect the removal of the ubiquitinated substrates in the proteasome-containing foci: inhibition of p97 activity resulted in an increase of $\sim 50 \%$ in the foci size. The role of UBE3A has remained elusive.

Taken together, the study unravels yet another mechanism by which the UPS copes with a proteotoxic cue. It also highlights RAD23B's pivotal role in the formation of the newly discovered, stress-induced transient proteasome-containing nuclear foci. The proteolytic activity of the proteasome at these foci is required for the rapid clearance of proteins, the ubiquitination of which was stimulated following acute stress. The rapid formation of proteolytically active proteasome-containing dense bodies can provide the cell with a defense mechanism to cope with various stresses by rapidly eliminating proteins that can otherwise generate aggregates.

\section{REFERENCES}

1. Turakhiya, A. et al. Mol. Cell 70, 906-919 (2018).

2. Feng, Z., Chen, X., Wu, X. \& Zhang, M. J. Biol. Chem. 294, 14823-14835 (2019).

3. Sun, D., Wu, R., Zheng, J., Li, P. \& Yu, L. Cell Res. 28, 405-415 (2018).

4. Yasuda, S. et al. Nature 578, 296-300 (2020).

5. Tomko, R. J. \& Hochstrasser, M. Annu. Rev. Biochem. 82, 415-445 (2013).

6. Livneh, I., Cohen-Kaplan, V., Cohen-Rosenzweig, C., Avni, N. \& Ciechanover, A. Cell Res. 26, 869-885 (2016).

7. Bard, J. A. M. et al. Annu. Rev. Biochem. 87, 697-724 (2018).

8. Elsasser, S., Chandler-Militello, D., Müller, B., Hanna, J. \& Finley, D. J. Biol. Chem. 279, 26817-26822 (2004).

9. Rao, H. \& Sastry, A. J. Biol. Chem. 277, 11691-11695 (2002).

10. Seibenhener, M. L. et al. Mol. Cell. Biol. 24, 8055-8068 (2004). 